\title{
Buchbesprechung: „Gesprengte Institution" in der Bredouille. Die École Expérimentale de Bonneuil-sur-Marne im Spannungsfeld von Inklusion, Psychiatriekritik, Psychoanalyse und neoliberalen Veränderungen. Eine ethnografisch orientierte Dispositivanalyse
}

\author{
Pierre-Carl Damian Link
}

Link, Pierre-Carl Damian (2020). Buchbesprechung:

„Gesprengte Institution“ in der Bredouille. Die École Expérimentale de Bonneuil-sur-

Marne im Spannungsfeld von Inklusion, Psychiatriekritik, Psychoanalyse und neoliberalen Veränderungen. Eine ethnografisch orientierte Dispositivanalyse In: Gingelmaier, Stephan / Langer, Janet / Bleher, Werner / Fickler-Stang, Ulrike /

Dietrich, Lars / Herz, Birgit (Hg.)

ESE Emotionale und Soziale Entwicklung in der Pädagogik der Erziehungshilfe und bei Verhaltensstörungen 2. Jahrgang (2020). Heft 2,

Macht und Ohnmacht in der Pädagogik bei psychosozialen Beeinträchtigungen, S. 80-91, Bad Heilbrunn: Verlag Julius Klinkhardt

ISBN 978-3-7815-2380-7

\section{(c) (1) (2) (2)}

Die Publikation (mit Ausnahme aller Fotos, Grafiken und Abbildungen) ist veröffentlicht unter der Creative Commons-Lizenz: CC BY-NC-SA 4.0 International https://creativecommons.org/licenses/by-nc-sa/4.0/ 
Geoffroy, Miriam Anne

Gießen: Psychosozial-Verlag.

2019, 708 Seiten, 79,90€

ISBN-13: 978-3-8379-2856-3

Miriam Anne Geoffroy legt mit ihrer über 700 Seiten starken Doktorarbeit an der FU Berlin eine Publikation vor, die geradezu nach dem Austausch mit der sonderpädagogischen Disziplin Pädagogik bei Verhaltensstörungen ,schreit': "Gesprengte Institution « in der Bredouille. Vielversprechend erscheint die vorliegende Monografie dahingehend, da sie Verhaltensweisen des Subjekts und institutionellen Strukturen sowie dahinterliegende Machtverhältnisse aus der Perspektive psychoanalytischer Pädagogik und poststrukturalistischer Theorien analysiert, ohne Sonderpädagogin zu sein. So fehlt zwar explizit der spezifisch sonderpädagogische Blick, der sich aber implizit zwischen den Zeilen doch finden lässt.

Trotz des enormen Umfangs ermöglicht die Autorin der Leserschaft sprachlich einen leichten und guten Zugang zu hochkomplexen Sachverhalten. Am Beispiel einer Pariser Institution, der École Expérimentale de Bonneuil-sur-Marne, arbeitet sie heraus, welchen schwerwiegenden Veränderungen pädagogische und therapeutische Einrichtungen durch neoliberale Praktiken ausgesetzt sind, die ein zeitintensives Arbeiten am Konflikt zunehmend verunmöglichen. Geoffroy hat keine Angst vor klaren Worten, sondern benennt politische und gesellschaftliche Missstände klar, die pädagogische Handlungsmöglichkeiten durch Finanzierung, Evaluation, flexiblere Arbeitszeiten und Sicherheitsdiskurse zunehmend beschränken. Dabei verdeutlich sie, mit welchen Antinomien eine inklusive Einrichtung umzugehen hat.

Im Inklusionsdiskurs standen Förderschulen und Sondereinrichtungen lange unter einem Generalverdacht, dass sie sich ihre Subjekte, für die sie vorgibt da zu sein, durch Diagnosen und Förderkategorien selbst hervorbringt. In diesem Sinne galten Sondereinrichtungen als in besonderer Weise legitimierungsbedürftig und waren wirklich in der Bredouille. Der Wind dreht sich allmählich und man sucht nun danach, welche Impulse die Sonderpädagogiken in Theorie und Praxis für das Ziel einer zunehmend inklusiveren Gesellschaft beizutragen haben. Schwer und schwerst belastete Subjekte mit Verhaltensauffälligkeiten verweisen auf die Grenzen inklusiver Bemühungen, fordern diese ein und rebellieren gegen eine zunehmende Unsichtbarkeit im Inklusionsdiskurs, in dem gerade diese Klientel zu verschwinden droht. Im ersten Kapitel führt Geoffroy auf die Institution Bonneuil als einen „Ort zum Fragen“ hin, erläutert den Aufbau und ihre Thesen. Dabei zeigt sie auf, dass eine inklusive Einrichtung permanenter wissenschaftlicher Begleitung bedarf, als eine Hinterfragung eigener Macht/ Ohnmacht-Positionen. 
Das zweite Kapitel beinhaltet die Frage des Forschungszugangs, der Methodologie und Methode einer ethnografisch orientierten Dispositivanalyse, die neue, vielversprechende Implikationen für sonderpädagogische Wissenschaftszugänge, im Hinblick auf Analyseraster für Macht- und Ohnmachtsdynamiken, bereithält. Besonders beeindruckt die systematisch klare und ausführliche Beschreibung der Forschungsmethodik, die die Verbindung eines ethnografischen Zugangs und der Dispositivanalyse sowie den Umgang mit besonders heterogenen Daten für Dritte nachvollziehbar gestaltet. Im dritten Kapitel wird die historische Entstehungsgeschichte, Kontext, Struktur, Konzept und Praxis der gesprengten Institution vorgestellt und die Einflüsse durch Antipsychiatrie und Psychoanalyse verdeutlicht. Poststrukturalistische Theorieansätze zur Analyse der gesprengten Institution stellt die Autorin im vierten Kapitel vor, bevor sie damit im fünften Kapitel die Problemsituationen psychosozialer Institutionen anhand der Aspekte Finanzierung, Evaluation und Akkreditierung und Teamarbeit aufzeigt und hinterfragt.

Kapitel sechs behandelt die Kritik an Geschlechterkonstruktionen, die eine Auseinandersetzung mit aktuellen psychoanalytischen Diskursen mehr berücksichtigen hätte können.

Die Innovationen, die Geoffroy in Bonneuil ausfindig macht, können auf sonderpädagogische Einrichtungen übertragen werden. Im abschließenden siebten Kapitel entwirft sie Mannonis Konzept der "gesprengte Institution“ als Modell und spricht mit ihr von einer „außerordentlichen Erfindung“ (S. 641), benennt dabei aber stets realistisch und ehrlich die Schwierigkeiten in und mit denen eine solche inklusive Einrichtung (bewusst) umzugehen wissen muss.

Neu an Geoffroys Studie, aber auch passend in die Phase des derzeitigen Inklusionsdiskurses, ist, dass sie der Leserschaft eine inklusive und psychoanalytisch-pädagogische Einrichtung vorstellt, die bisher kaum bis gar nicht wissenschaftliche Rezeption im deutschsprachigen Raum erfahren hat. Positionen innerhalb der Erziehungswissenschaften verstehen Inklusion als dritte Welle der Antipsychiatriebewegung. Auch Geoffroy arbeitet anhand einer inklusiven Einrichtung heraus, wie sehr die Konzeption "gesprengter Institution“ von Antipsychiatrie und strukturaler Psychoanalyse geprägt ist. Viele Sondereinrichtungen sind bereits jetzt „gesprengte Institutionen “ und dennoch könnte die Sonderpädagogik sehr von diesem Konzept profitieren, das versucht, „zwischenmenschliche Beziehungen auf institutionelle Strukturen“ zu übertragen (S. 21). Möchte eine Einrichtung wirklich inklusiv sein, dann tut sie gut daran, sich dieser Sichtweise zu bedienen, allen voran wenn man bedenkt, dass Inklusion zunächst ein konflikthaftes Geschehen darstellt.

Besonders genau arbeitet die Autorin auch historische Entwicklungen von Bonneuil heraus, die seit ihrer Gründung durch Maud Mannoni vor 50 Jahren (1969) stattgefunden haben und verknüpft diese mit einem umfassenden interdisziplinären Theoriekorpus.

Für die Sonderpädagogik dürfte Kapitel 7.3 von besonderer Relevanz sein. Geoffroy überträgt dort ihre Thesen auf Diskurse in Deutschland, allen voran auf den Inklusionsdiskurs (S. 677-81). Kritisch zu diskutieren ist hierbei die Leistungsdimension von Erziehung, sowie die Vergabe von Diagnosen und Förderbedarfen. Geoffroys Kritik, dass die Vergabe eines Förderbedarfs als „Etikett“ zu verstehen sei, dass nur dazu diene an zusätzliche Ressourcen zu kommen (S. 677), ist doch etwas zu kurz gedacht, und wird v.a. nicht, was eigentliches Anliegen der Arbeit ist, vom Subjekt aus gedacht. Negative Auswirkungen von Diagnosen werden stellenweise leider undifferenziert in den Blick genommen (S. 678). Diagnostik sei Etikettierung und mit Diskriminierung verbunden, so „dass die betroffenen Kinder daran 
‘zersprengen` können“ (S. 678) und ein „symbiotisches Abhängigkeitsverhältnis“ zwischen Kind und Diagnose vorliege (S. 678).

Geoffroy trägt aber die vielleicht berechtigte Sorge vor, dass die Existenz der Schulen künftig von der Anwesenheit diagnostizierter Kinder abhänge (S. 678), bei Psychiatrien sieht sie das schon als gegeben. So ist doch die Frage zu stellen, ob die Psychiatrien und Fördereinrichtungen nicht gerade deshalb da sind, weil es Subjekte gibt, die an den Umständen, Beziehungen und an sich selbst leiden, dass es seitens der Anderen wirklich eine ethisch begründete Fürsorge- und Erziehungspflicht gibt, und Sondereinrichtungen temporär als Durchgangsorte wirklich für leidende Subjekte da sind und sein müssen.

Zusammenfassend kann man sagen, dass es der Autorin vorwiegend um die Verbindungen und Wechselwirkungen von gesellschaftlichen Macht- und Herrschaftsstrukturen, bestimmter Institutionen, alltäglicher Praktiken und normativer Diskurse gehe (S. 65). Geoffroy ist in der Diskussion ihrer Ergebnisse sympathisch bescheiden und verweist auf eine Wahrheit im Erziehungsgeschehen, die nicht in Vergessenheit geraten darf:

Die Tatsache, dass alle Kinder andere sie anerkennende Subjekte für ihre Subjektwerdung benötigen, ist keine neue Erkenntnis. Dass auch Menschen, die sich in Schwierigkeiten befinden und in unserer Gesellschaft meistens ausgeschlossen [...] werden, andere anerkennende Subjekte benötigen, um sich als Subjekte (wieder) zu (re-) konstituieren, stellt ebenfalls keine neue Erkenntnis dar. (S. 675)

Insofern leistet die Autorin wirklich nicht nur „einen (kleinen) Beitrag für den komplexen Kampf gegen Diskriminierungen und Ausgrenzungen“ (S. 675f.), sondern einen entsprechend großen. Sie schlägt vor pädagogische „Handlungsspielräume zu erweitern oder zumindest noch vorhandene besser zu verteidigen, bevor es zu spät wird“ (S. 676). Für alle besonderen Einrichtungen gilt auch in inklusiven Zeiten: „Keine Abschaffung, sondern Sprengung“ (S. 189). Mit Sprengung der Institution meint Geoffroy die „Entschleierung von pathologisierenden und sklerotisierenden Funktionen“ (S. 7). Letztlich geht es der Autorin bei ihrer Reflexion von Macht-Ohnmachtdynamiken sozialer Institutionen, ihrer Akteure und adressierten Subjekte um eine apersive, also eine öffnende, durchlässigere Funktion besonderer Einrichtungen. Fördereinrichtungen können, insofern sie es nicht jetzt schon sind, zu konstitutiven Orten für Subjekte in Schwierigkeiten werden, die sich der kollektiven Verantwortung füreinander nicht entziehen, und die Subjektwerdung aller beteiligten zu ermöglichen suchen, als Durchgangsorte zum Leben und zum Fragen (S. 19). 